Article

\title{
Superhydrophobic Composite Coating with Excellent Mechanical Durability
}

\author{
Cheng Ke ${ }^{1,2}$, Yifan Fang ${ }^{1,2}$, Zheng Zhou ${ }^{3}$, Guohong Wang ${ }^{1,2}$, Yongjia Liu ${ }^{2}$, Wei $\mathrm{Wu}^{1,2}$, Lishuang Xiao ${ }^{2}$, \\ Mengzhuo Zhang ${ }^{4}$, Haibao $\mathrm{Hu}^{4}$ and Jianxi Liu ${ }^{1,2, *(1)}$
}

check for

updates

Citation: Ke, C.; Fang, Y.; Zhou, Z.; Wang, G.; Liu, Y.; Wu, W.; Xiao, L.;

Zhang, M.; Hu, H.; Liu, J.

Superhydrophobic Composite

Coating with Excellent Mechanical

Durability. Coatings 2022, 12, 185

https://doi.org/10.3390/

coatings12020185

Academic Editor: Robert J. K. Wood

Received: 31 December 2021

Accepted: 29 January 2022

Published: 31 January 2022

Publisher's Note: MDPI stays neutral with regard to jurisdictional claims in published maps and institutional affiliations.

Copyright: (C) 2022 by the authors. Licensee MDPI, Basel, Switzerland. This article is an open access article distributed under the terms and conditions of the Creative Commons Attribution (CC BY) license (https:// creativecommons.org/licenses/by/ $4.0 /)$.
1 Research \& Development Institute, Northwestern Polytechnical University in Shenzhen, Shenzhen 518057, China; chengke@mail.nwpu.edu.cn (C.K.); fyfasd123@163.com (Y.F.); wgh@mail.nwpu.edu.cn (G.W.); weiwu95@mail.nwpu.edu.cn (W.W.)

2 State Key Laboratory of Solidification Processing, Center of Advanced Lubrication and Seal Materials, Northwestern Polytechnical University, Xi'an 710072, China; 2018303505@mail.nwpu.edu.cn (Y.L.); xiaolishuang@mail.nwpu.edu.cn (L.X.)

3 Department of Fluid Mechanics, Northwestern Polytechnical University, Xi'an 710072, China; 15100241888@163.com

4 School of Marine Science and Technology, Northwestern Polytechnical University, Xi'an 710072, China; zhangmzhuo@mail.nwpu.edu.cn (M.Z.); huhaibao@nwpu.edu.cn (H.H.)

* Correspondence: jianxiliu@nwpu.edu.cn

\begin{abstract}
Superhydrophobic surfaces have great potential for self-cleaning, anti-icing, and dragreducing because of their water repellency property. However, their super-hydrophobicity is destroyed under mechanical abrasion due to the vulnerability of the delicate surface textures. Here, we demonstrate a strategy to create a robust superhydrophobic surface using MXene and fluoridated silica as functional fillers in epoxy resin. The fluoridated silica produces low surface energy, MXene serves as a wear-resistant phase and epoxy resin is the binding matrix. The composite coating demonstrates a self-cleaning effect to remove particles from the superhydrophobic surface by rolling water droplets. Moreover, the coating exhibits excellent mechanical durability by standing abrasion to maintain super-hydrophobicity. The superhydrophobic composite coating has the advantages of low cost and feasibility and has the potential for expandable industrial promotion.
\end{abstract}

Keywords: superhydrophobic; self-cleaning; $\mathrm{SiO}_{2}$; Mxene; epoxy resin; spraying

\section{Introduction}

Superhydrophobic surface, inspired by the "Lotus effect" (contact angle $>150^{\circ}$, sliding angle $<10^{\circ}$ ), has received considerable attention in the fields of self-cleaning, anti-icing, antifog, and anticorrosion. Lotus leaves have highly water repellency due to their surface papillary structure and epidermal wax crystals [1-4]. Understanding the various wetting transitions (WT) in the sense of their basic mechanism is crucial for the manufacture of superhydrophobic surfaces, from Young's equation, suitable for ideal conditions, to the Wenzel model, with roughness factor correction. The Cassie model, which is further combined with the influence of the chemical properties of the material surface, completes the wetting theory and has theoretical guiding significance for the experiment [5]. Currently, superhydrophobic surface design is mainly based on low surface energy and micro/nanostructures [5-7]. Methods for preparing rough surface structures can be divided into two categories. One is the top-down approach that introduces fine structures to generate numerous cavities. Deng et al. prepared armored superhydrophobic surfaces and interconnected networks by photolithography, which had impressive robustness in protecting the internal nanoscale structure [7]. Disadvantages of the method include high costs, complex operations, and a lack of general promotion. The other is the "bottom-up" approach that begins with a single small component to produce a complex surface, such as 
spraying, spin coating, and dipping physical deposition techniques, to prepare superhydrophobic coatings [6,8-11]. This method incorporates a viscous resin solution as the matrix and functional powders as the reinforcement to prepare a superhydrophobic composite coating. Due to the advantage of feasibility and low cost, the spray-coating method shows manufacturing potential for industrial application.

Nanomaterials are widely used to construct superhydrophobic composite coatings due to their specific surface chemistry and customized functionality [12-15]. By taking advantage of interface interaction between the nanomaterials and the matrix, the inherent defects, such as the microcracks and pores of the polymer-based coating, can be repaired $[16,17]$. The random micro/nanostructures are generated by solvent volatilization during the spraying and curing process of precursor solution with the inclusion of nanomaterials, which are successfully constructed to prepare superhydrophobic composite coatings. Additionally, the composite coating possesses more functionality due to the customized functions of various nanomaterials. Among these, $\mathrm{SiO}_{2}$ nanoparticles are often used to produce superhydrophobic coatings that can improve the anti-aging and chemical resistance of polymer coatings [18-20]. For example, Wang et al. prepared a transparent superhydrophobic coating by using a OSP scraping ink bar to control the thickness of fluoro-silane-modified $\mathrm{SiO}_{2}$ nanoparticles deposited on PDMS [19]. However, such superhydrophobic coatings have nanoparticles on the surface and generally show poor wear resistance, because the rough structure can be destroyed under mechanical wear. In addition, MXene, as a new member of the two-dimensional nanomaterial family, has exhibited excellent solid lubricating properties due to interlayer displacement of the weakly bonded multilayer structure [21,22]. However, introducing MXene to create a robust superhydrophobic coating is still challenging because the balance between super-hydrophobicity and abrasion resistance needs to be regulated.

In this paper, a robust self-cleaning superhydrophobic composite coating is spraycoated onto substrates by using MXene flakes and fluoridated $\mathrm{SiO}_{2} \mathrm{NPs}\left(\mathrm{F}-\mathrm{SiO}_{2}\right)$ and as reinforcements in epoxy resin. Hydrophobic $\mathrm{SiO}_{2} \mathrm{NPs}$ were prepared by $1 \mathrm{H}, 1 \mathrm{H}, 2 \mathrm{H}$, 2H-perfluorodecyltriethoxysilane (PFDTES) modification in order to have low surface energy. Then, the $\mathrm{F}-\mathrm{SiO}_{2} \mathrm{NPs}$ and $\mathrm{MXene}$ were introduced into epoxy resin to produce superhydrophobic composite coating. The MXene flakes and $\mathrm{F}-\mathrm{SiO}_{2}$ spheres in the composite coating provided higher roughness and more "air pockets". The epoxy resin was cross-linked to produce micro-protrusions and a stable network structure, which played a key role in protecting the interior cavity and encapsulated nanomaterials to maintain the high contact angle. The robustness of the coating was verified by abrasion experiments with 600-mesh silicon carbide sandpaper, and the super-hydrophobicity of the coating was maintained until 100 cycles of abrasion under a pressure of $5 \mathrm{kPa}$. The superhydrophobic composite coating shows excellent self-cleaning performance and mechanical durability.

\section{Materials and Methods}

\subsection{Materials and Chemicals}

$1 \mathrm{H}, 1 \mathrm{H}, 2 \mathrm{H}, 2 \mathrm{H}-$ Perfluorodecyltriethoxysilane (PFDTES, 96\%), $\mathrm{NH}_{3} \cdot \mathrm{H}_{2} \mathrm{O}(\mathrm{AR}, 25 \%$ 28\%), polyether-amine (D-400), silica nanoparticles (15 nm, 99.99\%) and lithium fluoride (LiF) were purchased from Aladdin Biochemical Technology. Titanium aluminium carbide $\left(\mathrm{Ti}_{3} \mathrm{AlC}_{2}, \sim 38 \mu \mathrm{m}\right)$ was purchased from 11 Technology Co., Ltd. (Jilin, China). Epoxy resin (E-44) was purchased from Xingchen Synthetic Material Co., Ltd. (Nantong, China). Concentrated hydrochloric acid $(\mathrm{HCl})$, ethanol and acetone were purchased from Shaanxi Dexiang Chemical. Deionized water $\left(>18 \mathrm{M} \Omega / \mathrm{cm}^{-1}\right)$ was obtained from an ultrapure water machine. All chemicals were used without further purification.

\subsection{Preparation of $\mathrm{Ti}_{3} \mathrm{C}_{2} \mathrm{~T}_{x}$ MXene Flakes}

Typically, $3.2 \mathrm{~g}$ of LiF was dispersed in $10 \mathrm{~mL}$ deionized water in a polytetrafluoroethylene beaker, followed by slowly adding $30 \mathrm{~mL} \mathrm{HCl}$. The polytetrafluoroethylene beaker was transferred to an oil bath, and $2 \mathrm{~g}$ of $\mathrm{Ti}_{3} \mathrm{AlC}_{2}$ was slowly added to the beaker under 
magnetic stirring to avoid violent exothermic activity in the solution. After continuous stirring and etching at $40{ }^{\circ} \mathrm{C}$ for $24 \mathrm{~h}$, the mixture was centrifuged at $3500 \mathrm{rpm}$ to remove the upper liquid and then washed with deionized water until the upper liquid was opaque. At this time, the upper liquid was collected until it retransmitted light. The collected upper liquid was centrifuged at a speed of 10,000 rpm, and the bottom sediment was collected, and then freeze-dried to obtain the MXene flakes.

\subsection{Preparation of PFDTES-Modified $\mathrm{SiO}_{2}$ Nanoparticles $\left(F-\mathrm{SiO}_{2}\right)$}

In a typical procedure, $1 \mathrm{~mL}$ of PFDTES, $90 \mathrm{~mL}$ of ethanol, $10 \mathrm{~mL}$ of DI water, and $4 \mathrm{~mL}$ of ammonia were stirred at $25^{\circ} \mathrm{C}$ for $2 \mathrm{~h}$ to promote the hydrolysis of siloxane. Subsequently, $2 \mathrm{~g} \mathrm{SiO}_{2}$ was added to react at $40{ }^{\circ} \mathrm{C}$ for $24 \mathrm{~h}$. Then, the solution was centrifuged, washed with ethanol three times, and dried overnight at $40{ }^{\circ} \mathrm{C}$. Finally, the $\mathrm{F}-\mathrm{SiO}_{2}$ was obtained by grinding.

\subsection{Preparation of the $\mathrm{F}-\mathrm{SiO}_{2} / \mathrm{MXene}$ Superhydrophobic Coating}

Scheme 1 depicts the process of fabricating the $\mathrm{F}_{-} \mathrm{SiO}_{2} / \mathrm{MXene}$ superhydrophobic coating by a spray-coating method. A Q235 steel sheet was polished by a series of sandpapers (80 \#, 400 \#, 800 \#) and sonicated in solvent (ethanol, acetone, and ethanol) to clean for $5 \mathrm{~min}$, and then blown dry with a nitrogen gun (HD-134, Dongguan, China). The subsequent oxygen plasma treatment for 3 min increased the surface energy and enhanced the bonding force between the steel substrate and the epoxy resin. Epoxy resin (E-44) and polyether-amine (D-400) were mixed at a mass ratio of 2:1, which was diluted with acetone and ultrasonically dispersed for $10 \mathrm{~min}$ to obtain paint with an epoxy resin concentration of $0.05 \mathrm{~g} \cdot \mathrm{mL}^{-1}$. Then $70 \mathrm{wt} . \%$ nanomaterials $\left(\mathrm{SiO}_{2}: \mathrm{MXene}=39: 1\right)$ were added and ultrasonically dispersed for $20 \mathrm{~min}$ to obtain a uniform solution. The $\mathrm{F}-\mathrm{SiO}_{2} / \mathrm{MXene}$ superhydrophobic coating was obtained by a spray-coating method with a spray gun; the spraying distance was $10 \mathrm{~cm}$, and the spraying pressure $0.05 \mathrm{MPa}$. Similarly, F-SiO $@_{2} @$ epoxy coating was prepared by using the same method, but only with $\mathrm{F}_{-} \mathrm{SiO}_{2}$ filler in resin. Finally, the spray-coated samples were cured at $80^{\circ} \mathrm{C}$ for $6 \mathrm{~h}$ and naturally cooled down to room temperature.

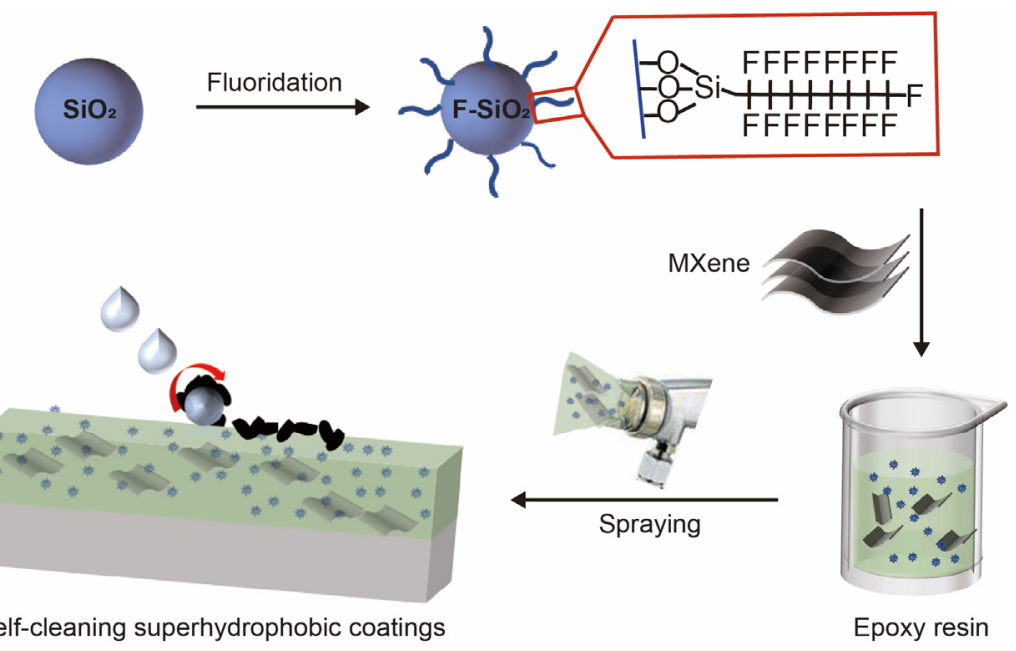

Scheme 1. Fabrication process of the $\mathrm{F}_{-} \mathrm{SiO}_{2} / \mathrm{MX}$ ene superhydrophobic coating.

\subsection{Characterization}

Morphology of $\mathrm{SiO}_{2}$ was obtained from field emission transmission electron microscopy (JEM-F200, JEOL, Tokyo, Japan) with accelerating voltages of $120 \mathrm{kV}$. Surface topography and EDS mapping of the coatings and MXene flakes were obtained from field emission scanning electron microscopy (Sigma 300, ZEISS, Oberkochen, Germany) with acceleration of 3 and $5 \mathrm{kV}$, respectively. The crystallinity and phase purity of MXene were 
tested by powder X-ray diffraction (Bruker II, Bruker, Karlsruhe, Germany) using $\mathrm{Cu}-\mathrm{K} \alpha$ radiation $(\lambda=1.54056 \AA)$ over $2 \theta$, ranging from $5^{\circ}$ to $80^{\circ}$ at $40 \mathrm{kV}$ and $40 \mathrm{~mA}$. The chemical structure of $\mathrm{SiO}_{2}$ was obtained from a Fourier transformation infrared spectrometer (FTIR, Bruker Tensor II, Bruker, Karlsruhe, Germany). The contact angle and sliding angle of the coating surface were measured by an optical contact angle meter (KRÜSS DSA, Hamburg, Germany) with $10 \mu \mathrm{L}$ of deionized water droplet each time. The droplet impact experiment was obtained with a high-speed camera (MotionXtru N4, IDT, Pasadena, CA, USA), $5 \mu \mathrm{L}$ of deionized water fell freely at a height of $1 \mathrm{~cm}$, and the impact image was captured at $2000 \mathrm{fps}$. The impact resistance and adhesion tests were obtained by the paint film impact tester (QCJ-50-100-120, Qixin, Tianjin, China), the cross-cut tester (QFH-A, Airuipu, Quanzhou, China), and 3M tape (Scotch 600, 3M, Saint Paul, MS, USA). Digital images were obtained by adding a macro lens to a mobile phone. Optical micrographs were obtained by optical microscopy (BX53, Olympus, Tokyo, Japan).

\section{Results and Discussion}

\subsection{Physicochemical Characterization of $\mathrm{SiO}_{2}$ Nanoparticles and MXene Flakes}

The inherent properties of $\mathrm{SiO}_{2}$ nanoparticles (NPs) and MXene flakes, such as morphology, chemical structure, and crystal structure, were investigated. Figure 1a shows transmission electron microscopy (TEM) images of the $\mathrm{SiO}_{2} \mathrm{NPs}$. The spherical $\mathrm{SiO}_{2} \mathrm{NPs}$ had an average size of $15 \mathrm{~nm}$. Scanning electron microscopy (SEM) images showed the morphology of MXene (Figure 1b,c), which had characteristically few layers and large size $(30-40 \mu \mathrm{m})$. Microscale MXene and nanoscale $\mathrm{SiO}_{2}$ are more conducive to constructing dual-scale micro/nanostructures of the composite coatings. The composition of $\mathrm{SiO}_{2} \mathrm{NPs}$ before and after PFDTES modification $\left(\mathrm{F}_{-} \mathrm{SiO}_{2}\right)$ was determined by FTIR spectroscopy (Figure 1d). $\mathrm{SiO}_{2} \mathrm{NPs}$ showed three characteristic peaks at 1103.6, 801.6, and $470.9 \mathrm{~cm}^{-1}$, corresponding to the antisymmetric stretching vibration absorption peak of $\mathrm{Si}-\mathrm{O}-\mathrm{Si}$ and the symmetric stretching vibration and bending vibration of $\mathrm{Si}-\mathrm{O}$. The broad peak at $3350-3500 \mathrm{~cm}^{-1}$ was a typical O-H group tensile vibration. The existence of hydroxyl groups on the surface of $\mathrm{SiO}_{2} \mathrm{NPs}$ can be used as active sites for surface grafting after hydrolysis of PFDTES. For the PFDTES-modified $\mathrm{SiO}_{2}$ nanoparticles, new peaks appeared at $1208.1,1148.0$ and $897.5 \mathrm{~cm}^{-1}$. These three peaks were attributed to the stretching vibrations of $-\mathrm{CF}_{2}$ and $-\mathrm{CF}_{3}[23,24]$, which proved the successful grafting of PFDTES on the surface of $\mathrm{SiO}_{2}$. Figure 1e shows the structural features of the $\mathrm{SiO}_{2} \mathrm{NPs}$ and $\mathrm{MXene}$ flakes that were characterized by XRD. For the $\mathrm{SiO}_{2}$, we observed the diffraction peak at $22.1^{\circ}$ that corresponded to the typical (111) plane [25]. Four diffraction peaks appeared at $7.05^{\circ}, 14.33^{\circ}, 28.75^{\circ}$, and $60.5^{\circ}$ corresponding to the (0002), (0004), (0008) and (110) plane of MXene, which were consistent with reports [26,27].

\subsection{Morphologies and Surface Wettability of the Coatings}

The rough structure of the material surface is an important factor in regulating wettability $[5,28]$, so we modulate the surface roughness of the coating by introducing different nanomaterials. Figure 2a-c show digital images of the pure epoxy resin coating (Figure 2a), $\mathrm{F}_{-} \mathrm{SiO}_{2} \mathrm{NPs}$ epoxy coating (Figure $2 \mathrm{~b}$ ), and $\mathrm{F}_{-} \mathrm{SiO}_{2} / \mathrm{MXene}$ epoxy coating (Figure 2c), and the inserts show the water contact angle on these coating surfaces. The pure epoxy resin coating had a contact angle of $89.7^{\circ} \pm 0.7^{\circ}$. By introducing the hydrophobic $\mathrm{F}_{-} \mathrm{SiO}_{2} \mathrm{NPs}$ in coating, the surface roughness of the coating was significantly increased, and the contact angle reached $146.6^{\circ} \pm 0.6^{\circ}$. Notable, large number of protrusions appear on the surface of the $\mathrm{F}-\mathrm{SiO}_{2} / \mathrm{MXene}$ epoxy coating, and the contact angle is $150.6^{\circ} \pm 0.4^{\circ}$. Thus, the surface roughness of the coating greatly improves the hydrophobicity of the nanoparticle-filled epoxy coating. 

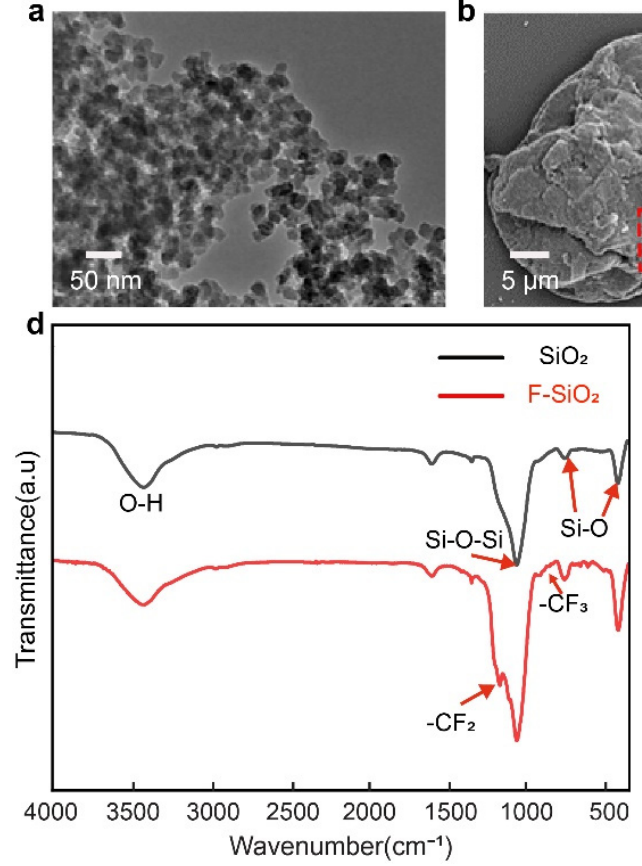
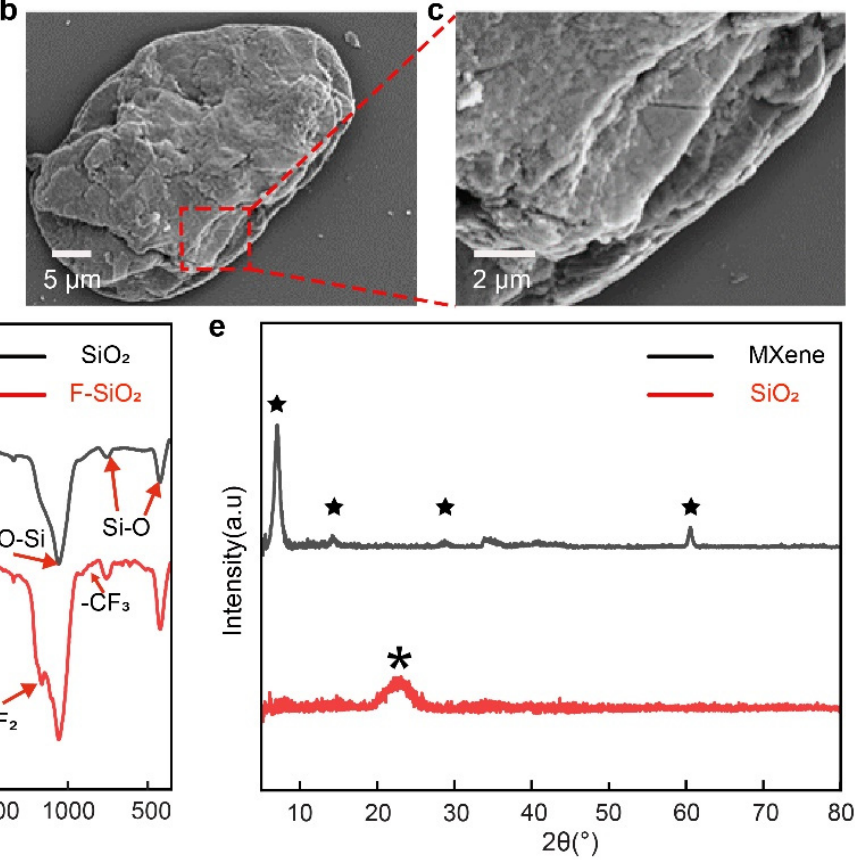

Figure 1. Characterization of the $\mathrm{SiO}_{2}$ nanoparticles and MXene flakes. (a) TEM image of the $\mathrm{SiO}_{2}$. (b-c) SEM image of the MXene flakes. (d) FT-IR spectra of the $\mathrm{SiO}_{2}$ and $\mathrm{F}-\mathrm{SiO}_{2}$. (e) XRD pattern of the $\mathrm{MXene}$ and $\mathrm{SiO}_{2}$, the symbols represent different diffraction peaks.

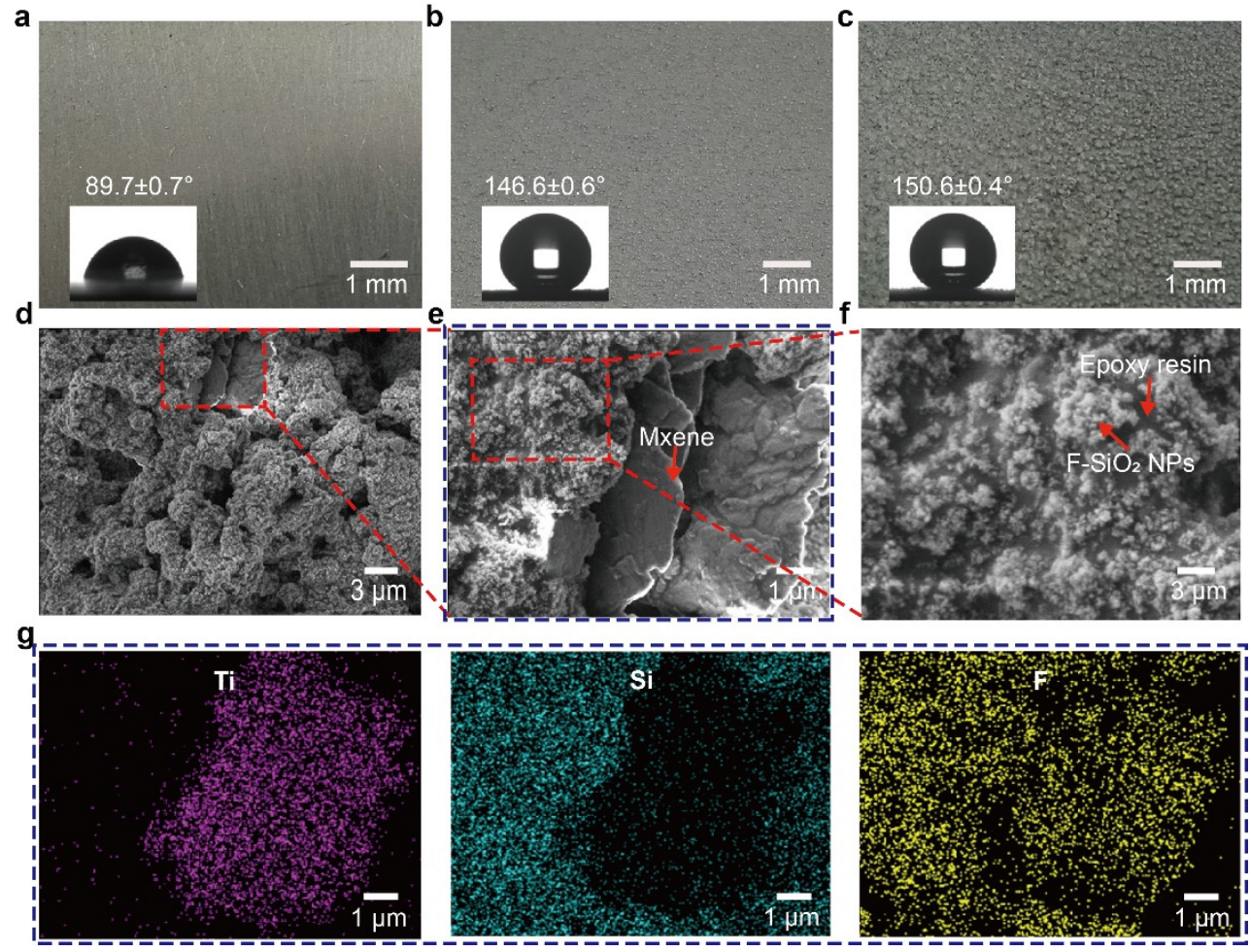

Figure 2. Surface morphology of the $\mathrm{F}_{-} \mathrm{SiO}_{2} / \mathrm{MX}$ ene superhydrophobic coating. (a-c) Digital images of epoxy resin coating, $\mathrm{SiO}_{2}$-embedded epoxy resin coating and $\mathrm{SiO}_{2} / \mathrm{MXene}$ superhydrophobic coatings. (d-f) SEM images of the $\mathrm{SiO}_{2} / \mathrm{MX}$ ene superhydrophobic coatings; the zoomed-in region shows details of the nanomaterials, (g) EDS mapping of $\mathrm{Ti}, \mathrm{Si}$, F elements on image (e) of the $\mathrm{SiO}_{2} / \mathrm{MXene}$ superhydrophobic coatings.

To further study the surface structure of the $\mathrm{F}_{-} \mathrm{SiO}_{2} / \mathrm{MXene}$ epoxy coating, we conducted SEM measurements (Figure 2d-f). We observed micro/nanostructures on the 
coating surface. Figure $2 \mathrm{~g}$ shows the EDS mapping of $\mathrm{Ti}, \mathrm{Si}$, and F elements of the superhydrophobic $\mathrm{F}_{-} \mathrm{SiO}_{2} / \mathrm{MXene}$ epoxy coating. The distribution of $\mathrm{Ti}$ in the EDS mapping image was consistent with the flake-like distribution of MXene in the SEM image. We proved that the $\mathrm{MXene}$ flakes and $\mathrm{F}-\mathrm{SiO}_{2} \mathrm{NPs}$ were partially exposed on the coating surface. Notably, we observed F element over the whole surface, originating from the migration of Fluoro-silane from $\mathrm{F}_{-} \mathrm{SiO}_{2}$ to the $\mathrm{MXene}$ during the high-temperature curing process for coating preparation [29]. The nanoscale hydrophobic $\mathrm{SiO}_{2} \mathrm{NPs}$ localized on the surface of microscale protrusions reduced the surface energy of the coating. Thus, the fluoridization together with micro/nanostructures introduced by nanomaterials is the key strategy for the preparation of a robust superhydrophobic coating.

\subsection{Self-Cleaning Test of Superhydrophobic Coating}

Self-cleaning is the most significant feature of superhydrophobic surfaces. The selfcleaning properties were tested by dripping water on the $\mathrm{F}_{-} \mathrm{SiO}_{2} / \mathrm{MXene}$ superhydrophobic coatings surface with contamination dust from nature. Figure $3 \mathrm{a}-\mathrm{c}$ show the process of the self-cleaning test when the superhydrophobic surface was inclined at $10^{\circ}$. The water droplets rolled off the coating and removed dust even at a very low inclination angle, showing excellent self-cleaning properties. Notably, only a small volume of water $(1-2 \mathrm{~mL})$ was used to clean the surface of $\mathrm{F}_{-} \mathrm{SiO}_{2} / \mathrm{MXene}$ coating.

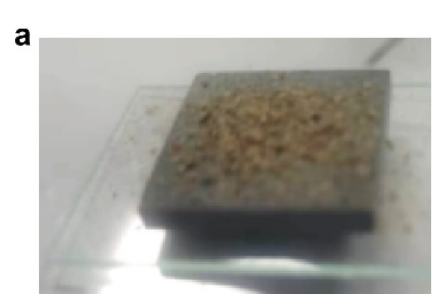

d

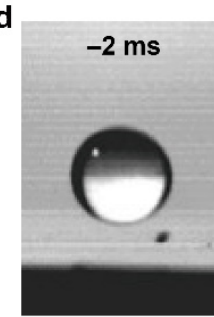

e

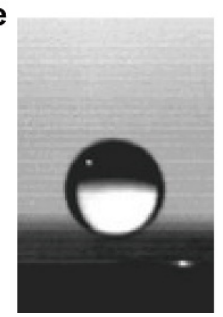

b

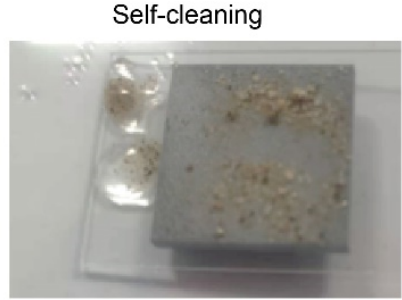

Epoxy resin
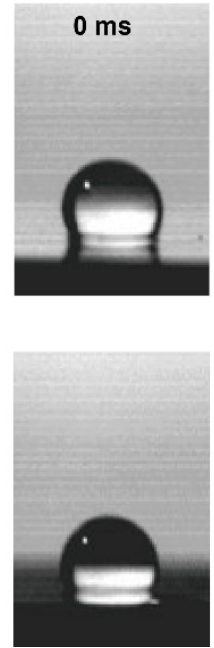
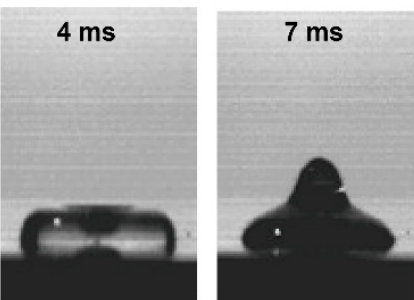

Superhydrophobic coatings
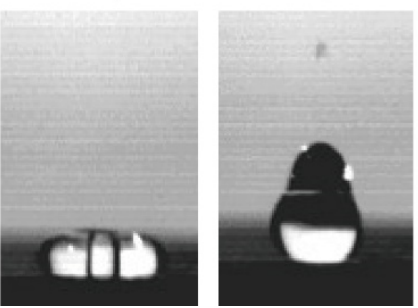
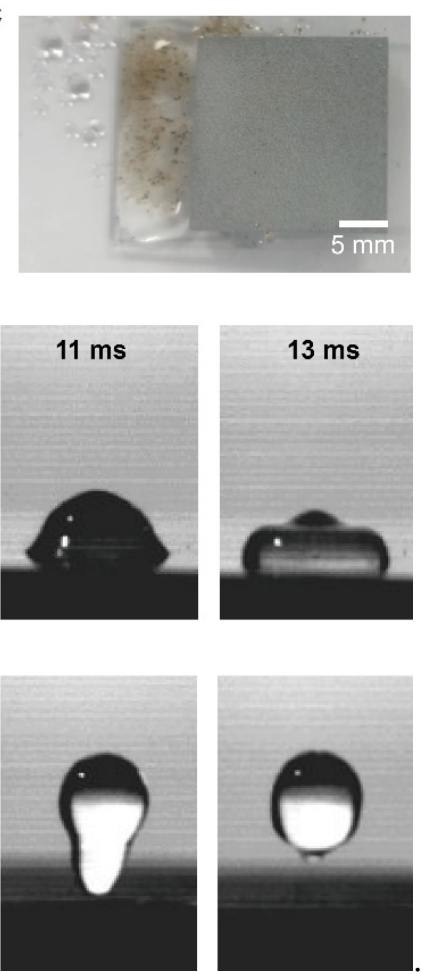

Figure 3. Self-cleaning of the $\mathrm{F}-\mathrm{SiO}_{2} / \mathrm{MXene}$ superhydrophobic coating. (a-c) Digital image of the coatings depict the self-cleaning test process by dripping water on a dust-containing superhydrophobic surface. Droplet impact experiment on (d) the pure epoxy resin coating and (e) the $\mathrm{F}-\mathrm{SiO}_{2} / \mathrm{Mxene}$ superhydrophobic coatings. Volume of the water droplet is $5 \mu \mathrm{L}$.

To further study the dynamics of droplet adhesion, we conducted droplet impact experiments. Figure $3 \mathrm{~d}$,e show the first rebound process of a $5 \mu \mathrm{L}$ water droplet, which fell freely from $1 \mathrm{~cm}$ above the epoxy resin coating (Figure 3d) and $\mathrm{F}_{-} \mathrm{SiO}_{2} / \mathrm{MXene}$ superhydrophobic coating (Figure 3e) and hit the surface. The droplet hit the epoxy resin coating and was unable to leave the surface because of the resistance of the surface adhesion to the bounce. In contrast, the droplet can rebound for the first time within $11 \mathrm{~ms}$ when 
hitting the $\mathrm{F}_{-} \mathrm{SiO}_{2} / \mathrm{MXene}$ superhydrophobic coating. The droplet shows typical rebound behavior after hitting the superhydrophobic surface, which first deformed and spread into a cake shape with the impact, then retracted and returned to a spherical shape. Notably, the droplet rebound time is positively correlated with the adhesion of the superhydrophobic surface $[30,31]$. Thus, the extremely low contact time between droplets and superhydrophobic surfaces proves the excellent liquid repellency, which can be widely used in self-cleaning and anti-icing fields.

\subsection{Robustness of Superhydrophobic Coating}

The anti-wetting ability of superhydrophobic surfaces depends on minimizing the solid-liquid interaction [5]. However, the destruction of the surface chemistry and rough structure causes fatal damage to the super-hydrophobicity. How to prepare a robust superhydrophobic coating is a key issue in meeting this challenge. An epoxy resin with strong adhesion was used as the adhesive, and $\mathrm{F}-\mathrm{SiO}_{2} \mathrm{NPs}$ and $\mathrm{MXene}$ flakes were used as fillers to prepare a superhydrophobic coating with excellent durability.

Figure 4 shows the abrasion test of the $\mathrm{F}_{-} \mathrm{SiO}_{2} / \mathrm{MXene}$ superhydrophobic coating on the effect of surface wettability. We firstly put water droplets with different $\mathrm{pH}$ values $(\mathrm{pH}=1,7$, and 13) on the surface of the coating (Figure $4 \mathrm{a})$. These droplets were all spherical and had no significantly differences from each other, which indicated robust liquid repellency for a wide range of $\mathrm{pH}$. Figure $4 \mathrm{~b}$ shows the sandpaper abrasion test to evaluate the mechanical durability of the coating. The $\mathrm{F}_{-} \mathrm{SiO}_{2} / \mathrm{MXene}$ epoxy coating surface was pushed horizontally and uniformly on 600 grit sandpaper for $10 \mathrm{~cm}$ under a pressure of $\sim 5 \mathrm{kPa}$, which was recorded as one cycle. After rubbing for 90 cycles, the contact angle of the coating stayed at about $150^{\circ}$, which did not change significantly with increasing abrasive paper abrasion distance (Figure $4 \mathrm{c}$ ). The contact angle showed a large fluctuation and decreased to about $142^{\circ} \sim 151^{\circ}$ at 100 cycles. However, the water droplets on the coating surface still maintained spherical shape after 100 times abrasion (Figure $4 \mathrm{~d}$ ). Notably, the droplets rolled off the sample edges, which proves that heavy abrasion had little influence on the self-cleaning performance. In addition, we noticed that part of the exposed metallic luster appeared on the optical microscope image, which meant that part of the coating was ground to show the surface of the substrate (Figure 4e). These results show that the contact angles have significant differences above and below $150^{\circ}$ after 100 times abrasion. We further conducted a water droplet impact experiment on the abrased $\mathrm{F}-\mathrm{SiO}_{2} / \mathrm{MXene}$ epoxy coating (Figure 4f). Compared with the droplet retraction process before abrasion, the rebound time was $11 \mathrm{~ms}$ on the abrased coating, which showed only a slight lag. Upon surface abrasion, our $\mathrm{F}_{-} \mathrm{SiO}_{2} / \mathrm{MXene}$ epoxy coating still had good liquid repellency, which is useful for practical application. The anti-wetting ability of superhydrophobic surfaces depends on minimizing the solid-liquid interaction [5]. However, the destruction of the surface chemistry and rough structure causes fatal damage to the super-hydrophobicity. How to prepare a robust superhydrophobic coating is a key issue for meeting this challenge. An epoxy resin with strong adhesion was used as the adhesive, and F-SiO 2 NPs and MXene flakes were used as fillers to prepare a superhydrophobic coating with excellent durability.

Figure 5 demonstrates the mechanism of abrasion resistance on the superhydrophobic $\mathrm{F}_{-} \mathrm{SiO}_{2} / \mathrm{MXene}$ composite coating. We observed the micro/nanostructures on the abrased coating from SEM image of the surface (Figure 5a). We found that large tracts of MXene are exposed to the outermost layer, while micro/nanostructures existed inside the coating. EDS mapping revealed the distribution of chemical elements after abrasion (Figure 5b-f). MXene was shown to be exposed to the surface of the coating due to the distribution of Ti elements coinciding with the SEM morphology (Figure 5b). The trace distribution of Si elements on the surface of $\mathrm{MXene}$ demonstrated the loss of $\mathrm{F}_{-} \mathrm{SiO}_{2}$ (Figure $5 \mathrm{c}$ ), which was the reason for the reduced water contact angle in some areas after abrasion. The distribution of $\mathrm{C}$ and $\mathrm{N}$ elements corresponded mainly to epoxy resin and polyether-amine curing agents, respectively (Figure 5d,e). The wide distribution of F elements contributed to the excellent liquid repellency of superhydrophobic coatings after abrasion (Figure 5f). Thus, 
the $\mathrm{F}_{-} \mathrm{SiO}_{2} / \mathrm{MXene}$ composite coating preserves the MXene flakes to resist abrasion and, the internal micro/nanostructures and wide distribution of low surface energy elements retain the excellent liquid repellency performance.
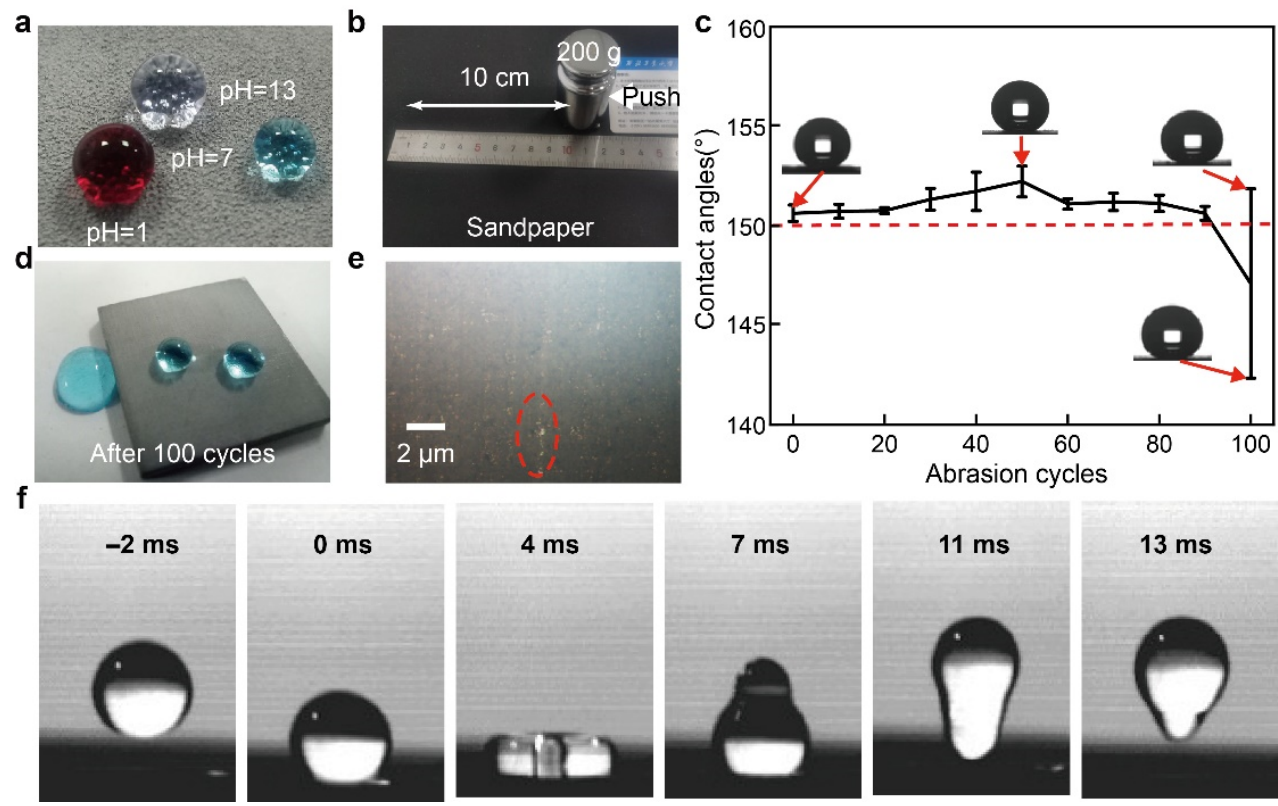

Figure 4. Robustness of the $\mathrm{F}-\mathrm{SiO}_{2} / \mathrm{MXene}$ superhydrophobic coating. (a) Digital images of water droplets with different $\mathrm{pH}$ values on superhydrophobic coatings. (b) Schematic of the abrasion test for superhydrophobic coatings. (c) The effect of abrasion cycles on the CA. (d-f) Digital images, optical microscope images, and droplet impact experiment images of the superhydrophobic coating after 100 abrasion cycles.
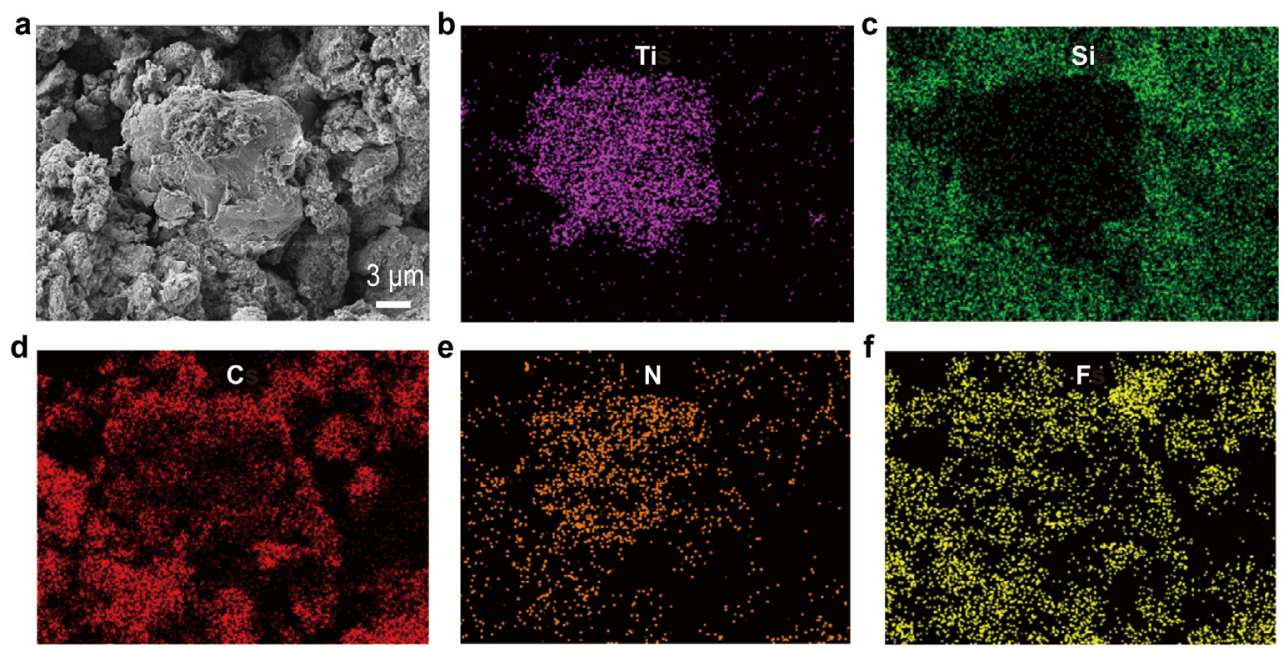

Figure 5. Surface morphology of the $\mathrm{F}-\mathrm{SiO}_{2} / \mathrm{MX}$ ene superhydrophobic coating after abrasion. (a) SEM images and (b-f) EDS mapping of the $\mathrm{F}_{-} \mathrm{SiO}_{2} / \mathrm{MXene}$ superhydrophobic coating after abrasion.

\subsection{Impact Resistance Test of Superhydrophobic Coating}

A hammer impact test was conducted to evaluate the adhesion of the coating to the substrate (Figure 6). Figure 6a shows a schematic diagram of the impact resistance test. The impact strength was defined as the maximum height at which a heavy hammer with a fixed weight of $1 \mathrm{~kg}$ falls on the test plate without damaging the coating. Figure $6 \mathrm{~b}, \mathrm{c}$ show the surface morphology of the coating after $\mathrm{H}=50 \mathrm{~cm}$ impact. We observed wrinkles on the coating surface, but without cracks or peeling. Obvious "impact pits" were observed in the digital image after $\mathrm{H}=70 \mathrm{~cm}$ impact (Figure $6 \mathrm{~d}$ ). We observe clear accumulation of 
impact edges from the optical microscope image (Figure 6e). The peeling of the coating also exposed the metal substrate, which proved that the coating was destroyed. Thus, the $\mathrm{F}_{-} \mathrm{SiO}_{2} / \mathrm{MXene}$ superhydrophobic composite coating has strong adhesion to the substrate, which can withstand external impact.

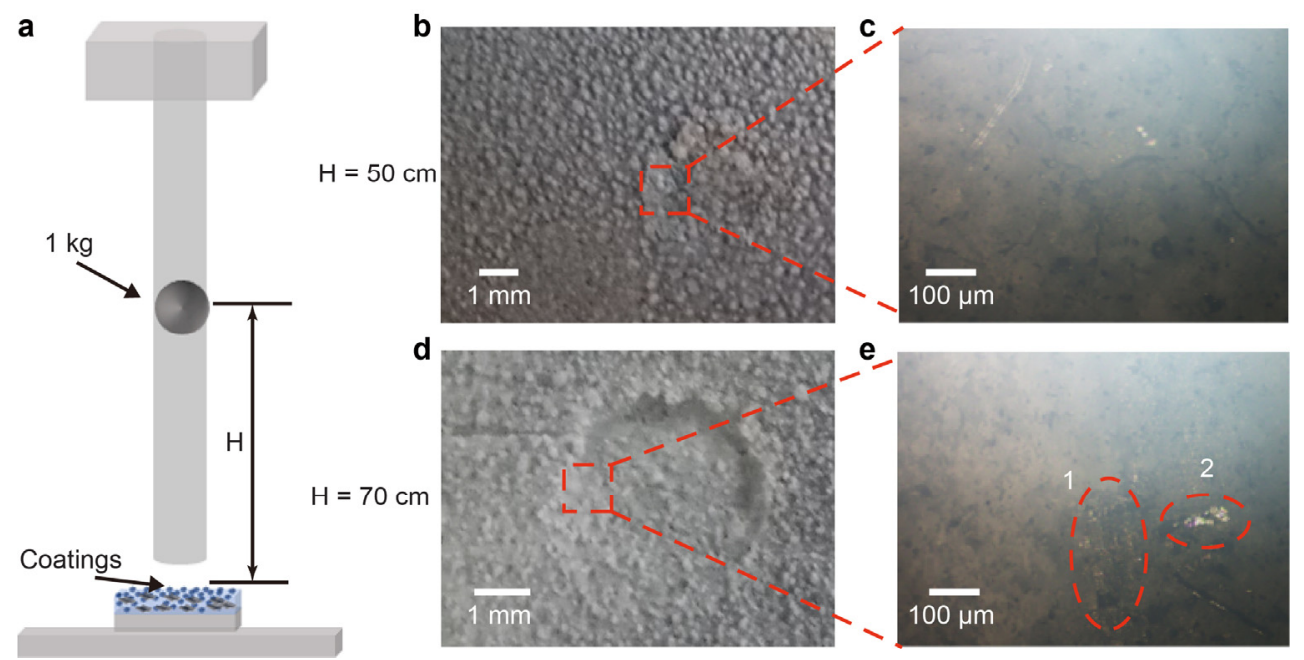

Figure 6. Hammer test showed the excellent impact resistance of the $\mathrm{F}_{-} \mathrm{SiO}_{2} / \mathrm{MXene}$ superhydrophobic coating. (a) Schematic of the impact resistance test for the superhydrophobic coatings. $(\mathbf{b}, \mathbf{c})$ Digital image and optical microscope image of the coating surface after impact testing at a height of $50 \mathrm{~cm}$. (d,e) Digital image and optical microscope image of the coating surface after impact testing at a height of $70 \mathrm{~cm}$.

\subsection{Cross-Cut Test of Superhydrophobic Coating}

One hundred grid knives manufactured in accordance with ISO 2409-1992 standards were used for coating adhesion determination. A blade with a cutter spacing of $1 \mathrm{~mm}$ cut the coating twice with horizontal force to obtain cross-cutting, perpendicular to each other. After cleaning with a soft brush, 3M tape was stuck onto the scratch and torn off at a small angle. Coating adhesion was measured by the area of tape adhesion and coating peeling off. Figure 7a shows digital images of the coating surface after the 100-grid knife test. A clear "cross-shaped" cutting path was observed on the surface of the coating, but no obvious peeling of the material from the substrate. A trace amount of white powder appeared on the $3 \mathrm{M}$ tape after peeling off the coating (Figure $7 \mathrm{~b}$ ). The optical microscope image showed the cutting path on the metal substrate (Figure 7c). However, only small pieces of the coating showed falling off, and the coating was kept as a whole protection membrane. These results indicate that the $\mathrm{F}_{-} \mathrm{SiO}_{2} / \mathrm{MXene}$ composite coating has excellent adhesion on the substrate. Thus, the $\mathrm{F}_{-} \mathrm{SiO}_{2} / \mathrm{MXene}$ superhydrophobic composite coatings have magnificent scratch resistance due to their strong adhesion to the substrate.
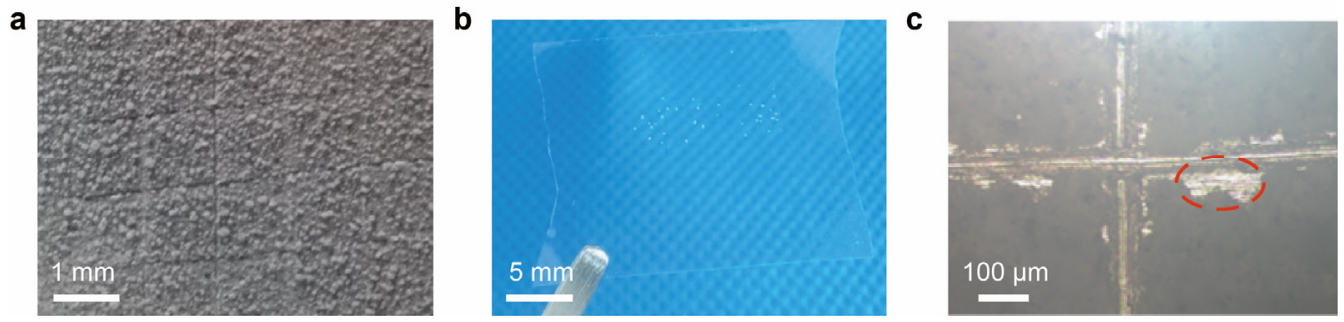

Figure 7. Cross-cut test showed strong adhesion of the $\mathrm{F}_{-} \mathrm{SiO}_{2} / \mathrm{MXene}$ superhydrophobic coating. Digital image of (a) the coating surface and (b) the $3 \mathrm{M}$ tape after the cross-cut test. (c) Optical microscope image of the coating surface after the cross-cut test. 


\section{Conclusions}

In summary, we demonstrated a universal strategy for preparing superhydrophobic composite coatings using $\mathrm{SiO}_{2}$ and $\mathrm{MXene}$ as reinforcements and epoxy resin as the matrix. The $\mathrm{F}_{-} \mathrm{SiO}_{2} / \mathrm{MXene}$ composite superhydrophobic coating revealed excellent self-cleaning performance due to the water repellency. The superhydrophobic coating had exceptional mechanical durability that could maintain high contact angle after wearing for 100 cycles under a pressure of $5 \mathrm{kPa}$. SEM images showed that MXene flakes maintained a large size and integrality in the resin that protected the super-hydrophobicity of internal F-SiO 2 NPs and improved the robustness. In addition, the first rebound of the water droplet impact was completed within $11 \mathrm{~ms}$, which was expected to be used in the field of anti-icing. The strong adhesion strength of the coatings was verified by hammer and cross-cut tests. Our work provides new ideas for robust superhydrophobic coating designs for applications in self-cleaning and anti-icing.

Author Contributions: Methodology, investigation and original draft preparation-C.K.; review and modify the manuscript-J.L.; supervision-H.H. and J.L.; investigation-Y.F., Z.Z., G.W., W.W., Y.L., L.X. and M.Z. All authors have read and agreed to the published version of the manuscript.

Funding: This research was funded by Guangdong Basic and Applied Basic Research Foundation (No.2021A1515410006), the National Natural Science Foundations of China (No.51879218); the Innovation Foundation for Doctor Dissertation of Northwestern Polytechnical University (No.CX2021060), National Undergraduate Training Programs for Innovation and Entrepreneurship (No.202110699121), and Funding for Innovation and Entrepreneurship of the Student Affairs Department of the Party Committee of Northwestern Polytechnical University (2021-cxcy-022).

Institutional Review Board Statement: Not applicable.

Informed Consent Statement: Not applicable.

Data Availability Statement: Not applicable.

Acknowledgments: We would like to thank the Analytical \& Testing Center of Northwestern Polytechnical University and Shaanxi Materials Analysis and Research Center.

Conflicts of Interest: The authors declare that there are no competing financial interests or personal relationships that appeared to influence the work reported in this paper.

\section{References}

1. Bhushan, B.; Jung, Y.C. Natural and biomimetic artificial surfaces for superhydrophobicity, self-cleaning, low adhesion, and drag reduction. Prog. Mater. Sci. 2011, 56, 1-108. [CrossRef]

2. Yoon, J.; Ryu, M.; Kim, H.; Ahn, G.N.; Yim, S.J.; Kim, D.P.; Lee, H. Wet-Style Superhydrophobic Antifogging Coatings for Optical Sensors. Adv. Mater. 2020, 32, 2002710. [CrossRef] [PubMed]

3. Chen, H.; Fan, H.; Su, N.; Hong, R.; Lu, X. Highly hydrophobic polyaniline nanoparticles for anti-corrosion epoxy coatings. Chem. Eng. J. 2021, 420, 130540. [CrossRef]

4. Barthlott, W.; Neinhuis, C. Purity of the sacred lotus, or escape from contamination in biological surfaces. Planta 1997, 202, 1-8. [CrossRef]

5. Zhang, W.; Wang, D.; Sun, Z.; Song, J.; Deng, X. Robust superhydrophobicity: Mechanisms and strategies. Chem. Soc. Rev. 2021, 50, 4031-4061. [CrossRef] [PubMed]

6. Goharshenas Moghadam, S.; Parsimehr, H.; Ehsani, A. Multifunctional superhydrophobic surfaces. Adv. Colloid Interface Sci. 2021, 290, 102397. [CrossRef] [PubMed]

7. Wang, D.; Sun, Q.; Hokkanen, M.J.; Zhang, C.; Lin, F.-Y.; Liu, Q.; Zhu, S.-P.; Zhou, T.; Chang, Q.; He, B.; et al. Design of robust superhydrophobic surfaces. Nature 2020, 582, 55-59. [CrossRef]

8. Cao, C.; Yi, B.; Zhang, J.; Hou, C.; Wang, Z.; Lu, G.; Huang, X.; Yao, X. Sprayable superhydrophobic coating with high processibility and rapid damage-healing nature. Chem. Eng. J. 2020, 392. [CrossRef]

9. Shen, Y.; Wu, Y.; Tao, J.; Zhu, C.; Chen, H.; Wu, Z.; Xie, Y. Spraying Fabrication of Durable and Transparent Coatings for Anti-Icing Application: Dynamic Water Repellency, Icing Delay, and Ice Adhesion. ACS Appl. Mater. Interfaces 2019, 11, 3590-3598. [CrossRef]

10. Archer, R.J.; Becher-Nienhaus, B.; Dunderdale, G.J.; Hozumi, A. Recent Progress and Future Directions of Multifunctional (Super)Wetting Smooth/Structured Surfaces and Coatings. Adv. Funct. Mater. 2020, 30, 1907772. [CrossRef] 
11. Yin, X.; Mu, P.; Wang, Q.; Li, J. Superhydrophobic ZIF-8-Based Dual-Layer Coating for Enhanced Corrosion Protection of Mg Alloy. ACS Appl. Mater. Interfaces 2020, 12, 35453-35463. [CrossRef] [PubMed]

12. Ahangaran, F.; Navarchian, A.H. Recent advances in chemical surface modification of metal oxide nanoparticles with silane coupling agents: A review. Adv. Colloid Interface Sci. 2020, 286, 102298. [CrossRef] [PubMed]

13. Zhu, X.; Yan, Q.; Cheng, L.; Wu, H.; Zhao, H.; Wang, L. Self-alignment of cationic graphene oxide nanosheets for anticorrosive reinforcement of epoxy coatings. Chem. Eng. J. 2020, 389, 124435. [CrossRef]

14. Zhou, H.; Wang, H.; Niu, H.; Gestos, A.; Wang, X.; Lin, T. Fluoroalkyl silane modified silicone rubber/nanoparticle composite: A super durable, robust superhydrophobic fabric coating. Adv. Mater. 2012, 24, 2409-2412. [CrossRef] [PubMed]

15. Wang, S.; Wang, Y.; Zou, Y.; Wu, Y.; Chen, G.; Ouyang, J.; Jia, D.; Zhou, Y. A self-adjusting PTFE/TiO 2 hydrophobic double-layer coating for corrosion resistance and electrical insulation. Chem. Eng. J. 2020, 402, 126116. [CrossRef]

16. Li, F.; Ma, Y.; Chen, L.; Li, H.; Zhou, H.; Chen, J. In-situ polymerization of polyurethane/aniline oligomer functionalized graphene oxide composite coatings with enhanced mechanical, tribological and corrosion protection properties. Chem. Eng. J. 2021, $425,130006$. [CrossRef]

17. Wu, B.; Lyu, J.; Peng, C.; Jiang, D.; Yang, J.; Yang, J.; Xing, S.; Sheng, L. Inverse infusion processed hierarchical structure towards superhydrophobic coatings with ultrahigh mechanical robustness. Chem. Eng. J. 2020, 387, 124066. [CrossRef]

18. Zheng, S.; Bellido-Aguilar, D.A.; Wu, X.; Zhan, X.; Huang, Y.; Zeng, X.; Zhang, Q.; Chen, Z. Durable Waterborne Hydrophobic Bio-Epoxy Coating with Improved Anti-Icing and Self-Cleaning Performance. ACS Sustain. Chem. Eng. 2019, 7, 641-649. [CrossRef]

19. Wang, P.; Chen, M.; Han, H.; Fan, X.; Liu, Q.; Wang, J. Transparent and abrasion-resistant superhydrophobic coating with robust self-cleaning function in either air or oil. J. Mater. Chem. A 2016, 4, 7869-7874. [CrossRef]

20. Ding, C.; Liu, Y.; Wang, M.; Wang, T.; Fu, J. Self-healing, superhydrophobic coating based on mechanized silica nanoparticles for reliable protection of magnesium alloys. J. Mater. Chem. A 2016, 4, 8041-8052. [CrossRef]

21. Grutzmacher, P.; Suarez, S.; Tolosa, A.; Gachot, C.; Song, G.; Wang, B.; Presser, V.; Mucklich, F.; Anasori, B.; Rosenkranz, A. Superior Wear-Resistance of $\mathrm{Ti}_{3} \mathrm{C}_{2} \mathrm{Tx}$ Multilayer Coatings. ACS Nano 2021, 15, 8216-8224. [CrossRef] [PubMed]

22. Marian, M.; Feile, K.; Rothammer, B.; Bartz, M.; Wartzack, S.; Seynstahl, A.; Tremmel, S.; Krauß, S.; Merle, B.; Böhm, T.; et al. $\mathrm{Ti}_{3} \mathrm{C}_{2} \mathrm{Tx}$ solid lubricant coatings in rolling bearings with remarkable performance beyond state-of-the-art materials. Appl. Mater. Today 2021, 25, 101202. [CrossRef]

23. Qing, Y.; Yang, C.; Yu, N.; Shang, Y.; Sun, Y.; Wang, L.; Liu, C. Superhydrophobic $\mathrm{TiO}_{2} /$ polyvinylidene fluoride composite surface with reversible wettability switching and corrosion resistance. Chem. Eng. J. 2016, 290, 37-44. [CrossRef]

24. Chen, H.; Wang, F.; Fan, H.; Hong, R.; Li, W. Construction of MOF-based superhydrophobic composite coating with excellent abrasion resistance and durability for self-cleaning, corrosion resistance, anti-icing, and loading-increasing research. Chem. Eng. J. 2020, 408, 127343. [CrossRef]

25. Song, S.; Yan, H.; Cai, M.; Huang, Y.; Fan, X.; Zhu, M. Constructing Mechanochemical Durable Superhydrophobic Composite Coating towards Superior Anticorrosion. Adv. Mater. Technol. 2021, 1-9. [CrossRef]

26. Wang, H.Y.; Sun, X.B.; Yang, S.H.; Zhao, P.Y.; Zhang, X.J.; Wang, G.S.; Huang, Y. 3D Ultralight Hollow NiCo Compound@MXene Composites for Tunable and High-Efficient Microwave Absorption. Nano-Micro Lett. 2021, 13, 1-15. [CrossRef]

27. Chang, M.; Jia, Z.; He, S.; Zhou, J.; Zhang, S.; Tian, M.; Wang, B.; Wu, G. Two-dimensional interface engineering of $\mathrm{NiS} / \mathrm{MoS}_{2} / \mathrm{Ti}_{3} \mathrm{C}_{2} \mathrm{Tx}$ heterostructures for promoting electromagnetic wave absorption capability. Compos. Part B 2021, $225,109306$. [CrossRef]

28. Lafuma, A.; Quere, D. Superhydrophobic states. Nat. Mater. 2003, 2, 457-460. [CrossRef]

29. Niu, W.; Chen, G.Y.; Xu, H.; Liu, X.; Sun, J. Highly Transparent and Self-Healable Solar Thermal Anti-De-Icing Surfaces When Ultrathin MXene Multilayers Marry Solid Slippery Self-Cleaning Coating. Adv. Mater. 2022. [CrossRef]

30. Nine, M.J.; Tung, T.T.; Alotaibi, F.; Tran, D.N.; Losic, D. Facile Adhesion-Tuning of Superhydrophobic Surfaces between "Lotus" and "Petal" Effect and Their Influence on Icing and Deicing Properties. ACS Appl. Mater. Interfaces 2017, 9, 8393-8402. [CrossRef]

31. Pan, R.; Zhang, H.; Zhong, M. Triple-Scale Superhydrophobic Surface with Excellent Anti-Icing and Icephobic Performance via Ultrafast Laser Hybrid Fabrication. ACS Appl. Mater. Interfaces 2021, 13, 1743-1753. [CrossRef] [PubMed] 